Correction

\title{
Correction: Xiang, Y.M.; Pan, W.F.; Jiang, H.B.; Zhu, Y.F.; Li, H. Measuring Software Modularity Based on Software Networks. Entropy 2019, 21, 344
}

\author{
Yiming Xiang ${ }^{1}$, Weifeng Pan ${ }^{2, *}$, Haibo Jiang ${ }^{2}$, Yunfang $\mathrm{Zhu}^{2}$ and $\mathrm{Hao}^{3}{ }^{3}$ \\ 1 School of Management and E-Business, Zhejiang Gongshang University, Hangzhou 310018, China; \\ futuretech@zjgsu.edu.cn \\ 2 School of Computer Science and Information Engineering, Zhejiang Gongshang University, \\ Hangzhou 310018, China; hbjiang88@163.com (H.J.); yunfangzj@163.com (Y.Z.) \\ 3 Department of Computer Science, Western Michigan University, Kalamazoo, MI 49008, USA; \\ hao.81.li@wmich.edu \\ * Correspondence: wfpan@zjgsu.edu.cn
}

The authors wish to make the following corrections to the paper [1].

On page 6 of [1], the last code line but one in Algorithm 1 (i.e., "18: $Q=$ sum_2m/sum_sigma;") should be updated to "18: $Q=$ sum_sigma/sum_2m;".

On page 9 of [1], the two numbers in the first line, 2.750 and 908.065 , should be replaced by 2.705 and 97.880 , respectively.

On page 13 of [1], the reference [8] should be replaced by the new reference [2] we listed below.

Note that the above errors do not affect the $Q$ values (see Tables 2 and 3 in [1]) that we computed for each subject software system in [1], since all the $Q$ values were computed according to the correct Equation (2) in [1]. Thus, the above changes will not affect the conclusions that we obtained in paper [1]. The authors would like to apologize for any inconvenience caused by these errors.

\section{References}

1. Xiang, Y.M.; Pan, W.F.; Jiang, H.B.; Zhu, Y.F.; Li, H. Measuring Software Modularity Based on Software Networks. Entropy 2019, 21, 344. [CrossRef]

2. Pan, W.F.; Ming, H.; Chang, C.K.; Yang, Z.J.; Kim, D.-K. ElementRank: Ranking Java Software Classes and Packages using Multilayer Complex Network-Based Approach. IEEE Trans. Softw. Eng. 2019. [CrossRef] 